\title{
GENERALIZED KÖTHE-TOEPLITZ DUALS
}

\author{
I.J. MADDOX \\ Department of Pure Mathematics \\ Queen's University of Belfast \\ Belfast BT7 1NN \\ Northern Ireland
}

(Received November 9, 1979)

ABSTRACT. The $\alpha$ and $\beta$-duals spaces of generalized $l_{p}$ spaces are characterized, where $0<p \leq \infty$. The question of when the $\alpha$ and $\beta$ dual spaces coincide is also considered.

KEY WORDS AND PHRASES. Generalized Köthe-Toeplitz dual spaces, Sequences of linear operators, Generalized $l_{p}$ spaces.

1980 MATHEMATICS SUBJECT CLASSIFICATION CODES. $40 \mathrm{CO5}, 40 \mathrm{J05}$.

1. INTRODUCTION.

$\mathrm{X}$ and $\mathrm{Y}$ denote complex Banach spaces with zero elements $\theta$, and $\|\cdot\|$ denotes the norm in either $\mathrm{X}$ or $\mathrm{Y}$. The continuous dual of $\mathrm{X}$ is written $\mathrm{X}^{*}$. By $s(X)$ we mean the space of all $X$-valued sequences $x=\left(x_{k}\right)$, where $x_{k} \in X$ for $k \in N=\{1,2,3, \ldots\}$. 
If $0<\mathrm{p}<\infty$, we mean by $\ell_{\mathrm{p}}(\mathrm{X})$ the space of all $\mathrm{X}$-valued sequences $x=\left(x_{k}\right)$ such that $\Sigma|| x_{k} \|^{p}<\infty$. Sums are over $k \in N$, unless otherwise indicated.

By $\ell_{\infty}(x)$ we denote the space of all $x=\left(x_{k}\right)$ such that sup ||$x_{k}||<\infty$.

In case $X=C$, the space of complex numbers, we write $\ell_{p}$ instead of $\ell_{\mathrm{p}}(\mathrm{C})$.

Let $A=\left(A_{k}\right)$ denote a sequence of linear, but not necessarily bounded, operators on $\mathrm{X}$ into $\mathrm{Y}$. If $\mathrm{E}$ is any nonempty subset of $\mathrm{s}(\mathrm{X})$ then the $\alpha$-dual of $E$ is defined to be

$$
E^{\alpha}=\left\{A: \Sigma|| A_{k} x_{k}||<\infty \text {, for all } x \in E\right\}
$$

The $\beta$-dual of $E$ is defined to be

$$
E^{B}=\left\{A: \sum A_{k} x_{k} \text { converges, for all } x \in E\right\}
$$

Since $\mathrm{Y}$ is complete, we have $\mathrm{E}^{\alpha} \subset \mathrm{E}^{\beta}$. The $\alpha$ and $\beta$ duals of $\mathrm{E}$ may be regarded as generalized Köthe-Toeplitz duals, since in case $X=Y=C$, when the $A_{k}$ may be identified with complex numbers $a_{k}$, the duals reduce to the classical spaces first considered by Köthe and Toeplitz [1].

Using the notation $(1 / p)+(1 / q)=1$, where $1 \leq p \leq \infty$, with the convention that $q=\infty$ when $p=1$, and $q=1$ when $p=\infty$, it is wel1-known that

$$
\ell_{\mathrm{p}}^{\alpha}=\ell_{\mathrm{p}}^{\beta}=\ell_{\mathrm{q}}
$$

We shall see that, in general, $\ell_{p}^{\alpha}(X) \subset e_{p}^{\beta}(X)$, where the inclusion may be strict. However, when $0<p \leq 1$ the $\alpha$ and $\beta$ duals coincide. Also, when $1<\mathrm{p} \leq \infty$, the $\alpha$ and $\beta$ duals coincide provided that $\mathrm{Y}$ is finite dimensional. 


\section{CHARACTERIZATION OF THE DUALS.}

THEOREM 1. Let $0<\mathrm{p} \leq 1$. Then $A \in \ell_{\mathrm{p}}^{\beta}(\mathrm{X})$ if and only if there exists $\mathrm{m} \in \mathrm{N}$ such that $\mathrm{A}_{\mathrm{k}}$ is bounded, for all $\mathrm{k} \geq \mathrm{m}$, and

$$
H=\sup _{k \geq m}|| A_{k}||<\infty .
$$

PROOF. Sufficiency. Let $(2.1)$ hold and $\Sigma|| x_{k} \|^{P}<\infty$. By a familiar inequality, see for example Maddox [2], page 22,

$$
\begin{aligned}
\left(\sum_{k=m}^{\infty}\left\|A_{k} x_{k}\right\|\right)^{p} & \leq \sum_{k=m}^{\infty}\left\|A_{k} x_{k}\right\|^{p} \\
& \leq \sum_{k=m}^{\infty}\left\|A_{k}\right\|^{p}\left\|x_{k}\right\|^{p} \\
& \leq H^{p} \sum|| x_{k} \|^{p} .
\end{aligned}
$$

Hence $\sum A_{k} x_{k}$ is absolutely convergent, and so convergent.

Necessity. Let $A \in \ell_{\mathrm{p}}^{\beta}(\mathrm{X})$ and suppose, if possible, that no such $m$ exists.

Then there are natural numbers $k(1)<k(2)<\ldots$ and $z_{i} \in \mathrm{x},\left\|z_{i}\right\| \leq 1$, such that for $\mathrm{i} \in \mathrm{N}$,

$$
|| A_{k(i)} z_{i} \|>i^{2 / p}
$$

Define $x_{k}=z_{i} / i^{2 / p}$ for $k=k(i)$ and $x_{k}=\theta$ otherwise. Then $x \in l_{p}(X)$ since $\Sigma|| x_{k} \|^{p} \leq \pi^{2} / 6$, but $\left\|A_{k} x_{k}\right\|>1$ for infinitely many $k$, contrary to the fact that $\sum \mathrm{A}_{\mathrm{k}} \mathrm{x}_{\mathrm{k}}$ converges.

Now suppose, if possible, that $\sup _{k \geq m}|| A_{k} \|=\infty$. Then there are natural numbers $k(1)<k(2)<\ldots$ with $k(1) \geq$ m such that for $i \in N$,

$$
\left\|A_{k(i)}\right\|>2 i^{2 / p}
$$

Choose $z_{i} \in X$ with $\left\|z_{i}\right\| \leq 1$ such that 2||$A_{k(i)} z_{i}\left\|\geq|| A_{k(i)}\right\|$, so by (2.3) 
we see that $(2.2)$ holds with the new $k(i)$ and $z_{i}$. We may define $x \in \ell_{p}(X)$ as above and obtain a contradiction. Hence (2.1) must hold, and the proof is complete.

If we examine the proof of Theorem 1 we see that in the sufficiency we had $\Sigma|| A_{k} x_{k} \|<\infty$, so that $A \in \ell_{p}^{\alpha}(X)$. Also, in the necessity, the constructions involved $x \in \ell_{p}(X)$ such that $\Sigma|| A_{k} x_{k}||$ was divergent. Hence we have:

THEOREM 2. If $0<p \leq 1$ then

$$
\ell_{p}^{\alpha}(x)=e_{p}^{\beta}(x)
$$

Next we consider the case $1<\mathrm{p}<\infty$.

THEOREM 3. Let $1<\mathrm{p}<\infty$. Then $A \in \ell_{\mathrm{p}}^{\alpha}(\mathrm{X})$ if and only if there exists $\mathrm{m} \in \mathrm{N}$ such that $\mathrm{A}_{\mathrm{k}}$ is bounded for all $\mathrm{k} \geq \mathrm{m}$, and

$$
M=\sum_{k=m}^{\infty}\left\|A_{k}\right\|^{q}<\infty .
$$

PROOF. Sufficiency. Let $(2.4)$ hold and $x \in \ell_{p}(X)$. By Holder's inequality,

$$
\sum_{k=m}^{\infty}\left\|A_{k} x_{k}\right\| \leq M^{1 / q}\left(\Sigma|| x_{k} \|^{p}\right)^{1 / p}<\infty .
$$

Necessity. Since $\ell_{p}^{\alpha}(x) \subset \ell_{1}^{\alpha}(x)$ when $p>1$, the existence of the $m$ in the theorem follows from Theorems 1 and 2 .

Now for $k \geq m$ we may choose $z_{k} \in X$ with $\left\|z_{k}\right\| \leq 1$ such that 2||$A_{k} z_{k}\|\geq\| A_{k} \|$.

For all $\lambda \in \ell_{p}$ we have $\left(\lambda_{k} z_{k}\right) \in \ell_{p}(X)$, so 


$$
\sum_{k=m}^{\infty}\left|\lambda_{k}\right||| A_{k} z_{k}||<\infty
$$

for all $\lambda \epsilon \ell_{\mathrm{p}}$. By $(1.1)$ it follows that

$$
H=\sum_{k=m}^{\infty}|| A_{k} z_{k} \|^{q}<\infty \text {, }
$$

whence $M \leq 2^{\mathrm{q}} \mathrm{H}$, so $(2.4)$ holds, and the proof is complete.

THEOREM 4. Let $1<\mathrm{p}<\infty$. Then $A \in \ell_{\mathrm{p}}^{\beta}(\mathrm{X})$ if and only if there exists $\mathrm{m} \in \mathrm{N}$ such that $\mathrm{A}_{\mathrm{k}}$ is bounded for all $\mathrm{k} \geq \mathrm{m}$, and

$$
\sup \sum_{k=m}^{\infty}|| A_{k}^{*} f||^{q}<\infty \text {, }
$$

where the supremum is over al1 $\mathrm{f} \in \mathrm{Y}^{*}$ with $\|\mathrm{f}\| \leq 1$.

PROOF. With the restriction that all the $A_{k}$ are bounded, and with different notation, this result was proved by Thorp [3]. Only the existence of $\mathrm{m}$ in the necessity needs attention, and this follows from Theorems 1 and 2 , and the fact that $\ell_{p}^{\beta}(X) \subset \ell_{1}^{\beta}(x)$.

Fina11y, we examine the case $p=\infty$. The proofs are left to the reader. We remark that with the restriction that all the $A_{k}$ are bounded, the result concerning $\ell_{\infty}^{\beta}(\mathrm{X})$ was given by Maddox [4].

THEOREM 5. A $\in \ell_{\infty}^{\alpha}(\mathrm{X})$ if and only if there exists $m \in N$ such that $A_{k}$ is bounded for al1 $\mathrm{k} \geq \mathrm{m}$, and

$$
\sum_{\mathrm{k}=\mathrm{m}}^{\infty}|| \mathrm{A}_{\mathrm{k}}||<\infty .
$$

THEOREM 6. A $\in \ell_{\infty}^{\beta}(X)$ if and only if there exists $m \in N$ such that $A_{k}$ is bounded for all $k \geq m$, and 


$$
\begin{aligned}
& \sup || \sum_{k=m}^{m+n} A_{k} x_{k} \|<\infty, \\
& \sup || \sum_{k=m}^{m+n} A_{k} x_{k} \| \rightarrow 0(m \rightarrow \infty),
\end{aligned}
$$

where the suprema are over all $\mathrm{n} \geq 0$ and all $\mathrm{x}_{\mathrm{k}} \in \mathrm{X}$ with $\left\|\mathrm{x}_{\mathrm{k}}\right\| \leq 1$.

\section{COINCIDENCE OF DUALS.}

It was shown in Theorem 2 that, when $0<p \leq 1, \ell_{p}^{\alpha}(X)=\ell_{p}^{\beta}(X)$ for any Banach spaces $\mathrm{X}$ and $\mathrm{Y}$.

We next shown that, when $1<p<\infty$, the inclusion $\ell_{p}^{\alpha}(x) \subset \ell_{p}^{\beta}(x)$ may be strict.

THEOREM 7. If $1<\mathrm{p}<\infty$ then there are Banach spaces $\mathrm{X}$ and $\mathrm{Y}$ such that $\ell_{\mathrm{p}}^{\alpha}(\mathrm{X}) \subset \ell_{\mathrm{p}}^{\beta}(\mathrm{X})$ with strict inclusion.

PROOF. Take $\mathrm{X}=\mathrm{Y}=\ell_{\mathrm{p}}$ and write

$$
e_{k}=(0,0, \ldots, 1,0,0, \ldots)
$$

where 1 is in the k-place and there are zeros elsewhere. Define bounded linear operators $A_{k}$ on $l_{p}$ into itself by

$$
A_{k} x=x_{k} e_{k}
$$

for each $x=\left(x_{k}\right) \in l_{p}$. Then $\left\|A_{k}\right\|=1$ for all $k \in N$, so $A$ is not in $l_{p}^{\alpha}(x)$ by Theorem 3 .

Let us now show that (2.5) holds. Take any $f \in \underset{\mathrm{p}}{\ell *}$ with $\|\mathrm{f}\| \leq 1$. Then for $x \in \ell_{p}$ we have

$$
f(x)=\Sigma f_{i} x_{i}
$$


for some $\left(f_{i}\right)$ such that $\Sigma\left|f_{i}\right|^{q} \leq 1$. Hence, by definition of $A_{k}^{*}$,

$$
\left(A_{k}^{*} f\right)(x)=f\left(A_{k} x\right)=f_{k} x_{k}
$$

and so ||$A_{k}^{*} f||=\left|f_{k}\right|$. Hence

$$
\Sigma|| A_{k}^{*} f||^{q}=\Sigma\left|f_{k}\right|^{q} \leq 1,
$$

so by Theorem 4 we have $A \in \ell_{p}^{\beta}(X)$.

Still with the case $1<\mathrm{p}<\infty$ we have:

THEOREM 8. If $1<\mathrm{p}<\infty$ and $\mathrm{Y}$ is finite dimensional then for any $\mathrm{X}$ we have

$$
\ell_{p}^{\alpha}(X)=\ell_{p}^{\beta}(X)
$$

PROOF. We have to show that $A \in \ell_{p}^{\beta}(X)$ implies $A \in \ell_{p}^{\alpha}(X)$. Now if $A \in l_{p}^{\beta}(X)$ then by Theorem 4 there exists $m \in N$ such that $A_{k}$ is bounded for all $k \geq m$. Suppose $Y$ has finite dimension $n$ and that $\left(b_{1}, b_{2}, \ldots, b_{n}\right)$ is a Hamel base for $\mathrm{Y}$. Then $\mathrm{y} \in \mathrm{Y}$ implies

$$
y=\sum_{i=1}^{n} \lambda_{i}(y) b_{i}
$$

where each $\lambda_{i} \in \mathrm{Y}^{*}$. Take $z \in \mathrm{X}$ and $\mathrm{k} \geq \mathrm{M}$. Then

$$
A_{k} z=\sum_{i=1}^{n} \lambda_{i}\left(A_{k} z\right) b_{i}
$$

and $\lambda_{i} \cdot A_{k} \in X^{*}$. Since $\sum_{k=m}^{\infty} A_{k} X_{k}$ converges for all $x \in \ell_{p}(X)$ we have

$$
\sum_{k=m}^{\infty}\left(\lambda_{i} \circ A_{k}\right) x_{k}
$$

convergent for all $x \in \ell_{p}(X)$ and each $i$. 
Choose $z_{k} \in X,|| z_{k} \| \leq 1$ such that $2\left|\left(\lambda_{i} \circ A_{k}\right) z_{k}\right| \geq|| \lambda_{i} \bullet A_{k}||$. If $t \in \ell_{p}$ then $\left(t_{k} z_{k}\right) \in \ell_{p}(X)$ so that

$$
\sum_{k=m}^{\infty} t_{k}\left(\lambda_{i} \cdot A_{k}\right) z_{k}
$$

converges for all $t \in \ell_{p}$, whence for each $i$,

$$
\sum_{k=m}^{\infty}\left\|\lambda_{i} \cdot A_{k}\right\|^{q}<\infty .
$$

By (2.9) and Hölder's inequality,

$$
\left\|A_{k}\right\|^{q} \leq \sum_{i=1}^{n}\left\|\lambda_{i} \cdot A_{k}\right\|^{q} \cdot\left(\sum_{i=1}^{n}\left\|b_{i}\right\|^{p}\right)^{q / p} .
$$

Denoting the final term in (2.11) by $\mathrm{H}$,

$$
\sum_{k=m}^{\infty}\left\|A_{k}\right\|^{q} \leq \mathrm{H} \sum_{i=1}^{n} \sum_{k=m}^{\infty}\left\|\lambda_{i} \cdot A_{k}\right\|^{q} .
$$

It follows from (2.10) and (2.12) that $(2.4)$ holds, so by Theorem 3 we have $A \in \ell_{p}^{\alpha}(X)$.

For certain values of $p$, and any $x$, the next result is the converse of Theorem 8.

THEOREM 9. If $2<\mathrm{p}<\infty$ and $\ell_{\mathrm{p}}^{\alpha}(\mathrm{X})=\ell_{\mathrm{p}}^{\beta}(\mathrm{X})$ then $\mathrm{Y}$ must be finite dimensional.

PROOF. Suppose, if possible, that $Y$ is infinite dimensional. Since $\mathrm{q}<2$, if $c_{k}=k^{-2 / q}$ then $\Sigma c_{k}<\infty$. By the Dvoretzky-Rogers theorem [5], there exists an unconditionally convergent series $\Sigma y_{k}$ in $Y$ such that $\left\|y_{k}\right\|^{2}=c_{k}$ for $k \in N$. Hence

$$
\Sigma|| y_{k}||^{q} \text { diverges. }
$$

Take $f \in X^{*}$ with $\|f\|=1$ and define rank one operators $A_{k}=y_{k} \otimes f$. Then $\left\|A_{k}\right\|=\left\|y_{k}\right\|$, so by (2.13) and Theorem 3, A is not in $l_{p}^{\alpha}(x)$. 
Now if $x \in \ell_{p}(X)$ then

$$
\sum A_{k} x_{k}=\Sigma f\left(x_{k}\right) y_{k}
$$

But $\left(f\left(x_{k}\right)\right) \in \ell_{\infty}$ and $\sum y_{k}$ is unconditionally convergent, so that $\sum f\left(x_{k}\right) y_{k}$ converges, whence $A \in \ell_{p}^{\beta}(X)$, which gives a contradiction.

We remark that it would appear that the argument of Theorem 9 cannot be used in the case $p=2$, since in a general Hilbert space $Y$ the unconditional convergence of $\Sigma y_{k}$ implies that $\Sigma|| y_{k} \|^{2}$.

However, we can deal with the case $p=2$ of Theorem 9 when $Y$ is a Hilbert space:

THEOREM 10. Let $Y$ be a Hilbert space and suppose $\ell_{2}^{\alpha}(X)=\ell_{2}^{\beta}(X)$. Then $\mathrm{Y}$ must be finite dimensional.

PROOF. Suppose, if possible, that $\mathrm{Y}$ is infinite dimensional. Choose an orthonormal sequence $\left(e_{k}\right)$ in $Y$ and denote the inner product in $Y$ by $\left(y_{1}, y_{2}\right)$. Take $g \in X^{*},|| g||=1$ and define rank one operators $A_{k}=e_{k} \otimes g$, so that $\| A_{k}||=1$. Now let $f \in Y *$ with $\| f|| \leq 1$. Then there exists y $\epsilon$ such that

$$
f(z)=(z, y)
$$

for all $z \in Y$, with $\|y\|=\|f\| \leq 1$. Then for $x \in X$,

$$
\left(A_{k}^{* f}\right)(x)=\left(g(x) e_{k}, y\right)=g(x)\left(e_{k}, y\right)
$$

Hence ||$A_{k}^{\star} f|| \leq\left|\left(e_{k}, y\right)\right|$, so by Besse1's inequality,

$$
\Sigma|| A_{k}^{* f}||^{2} \leq\|y\|^{2} \leq 1
$$

Thus (2.5) holds with $q=2$, and so $A \in \ell_{2}^{\beta}(X)$. But $A \ddagger \ell_{2}^{\alpha}(X)$ since ||$A_{k}||=1$ for a11 $k$. This contradiction implies our result. 
The case $p=\infty$ is due essentially to Thorp [3], who shows that $\ell_{\infty}^{\alpha}(X)=\ell_{\infty}^{\beta}(X)$ if and only if $Y$ is finite dimensional.

\section{REFERENCES}

1. Köthe, G. and Toeplitz, 0. Lineare Räume mit unendlich vielen Koordinaten und Ringe unendlicher Matrizen, J. reine angew. Math. 171 (1934), 193-226.

2. Maddox, I.J. Elements of Functional Analysis, Cambridge University Press, 1970.

3. Thorp, B.L.D. Sequential-evaluation convergence, J. London Math. Soc. 44 (1969), 201-209.

4. Maddox, I.J. Matrix maps of bounded sequences in a Banach space, Proc. American Math. Soc. 63 (1977), 82-86.

5. Dvoretzky, A. and Rogers, C.A. Absolute and unconditional convergence in normed linear spaces, Proc. Nat. Acad. Sci. (U.S.A.) 36 (1950) 192-197. 


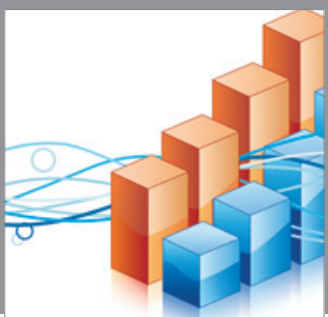

Advances in

Operations Research

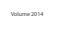

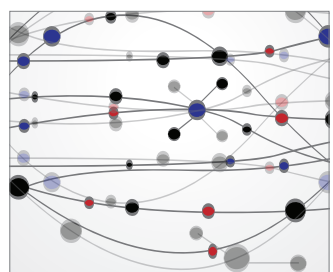

\section{The Scientific} World Journal
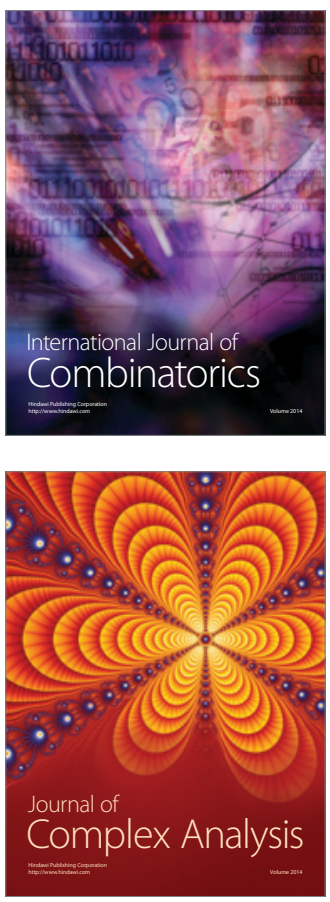

International Journal of

Mathematics and

Mathematical

Sciences
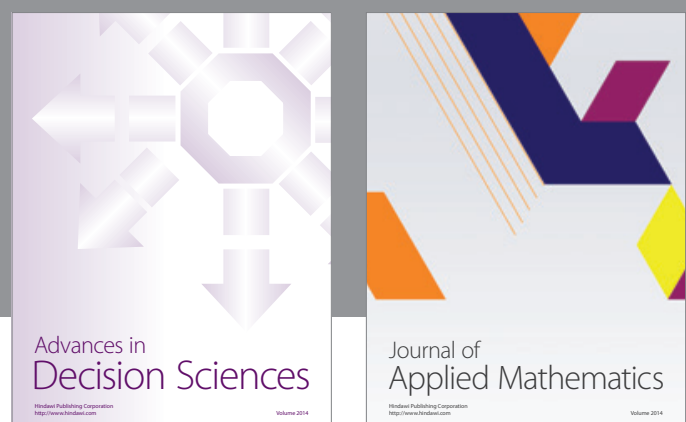

Journal of

Applied Mathematics
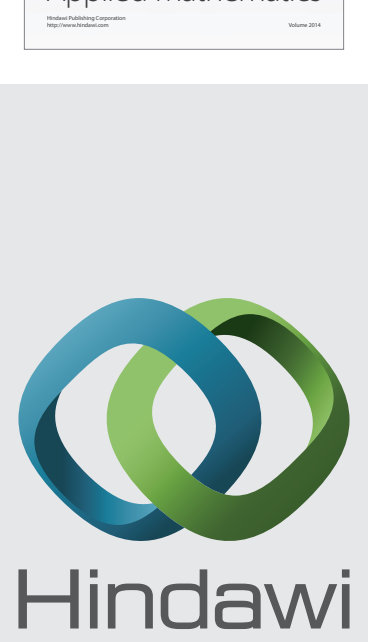

Submit your manuscripts at http://www.hindawi.com
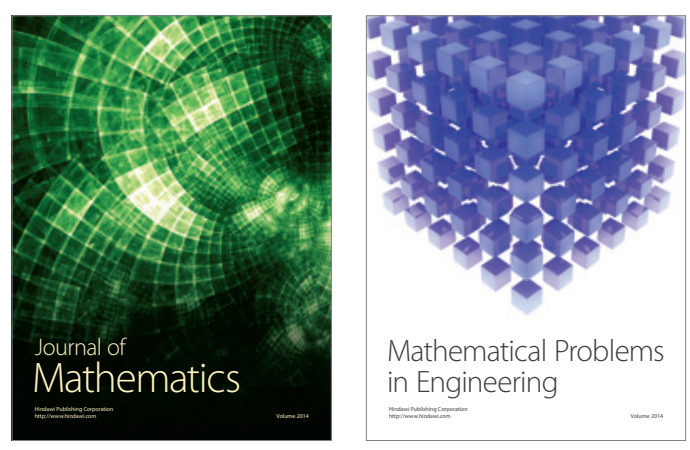

Mathematical Problems in Engineering
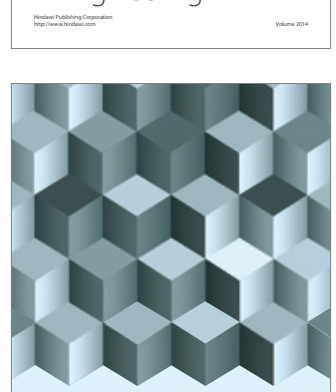

Journal of

Function Spaces
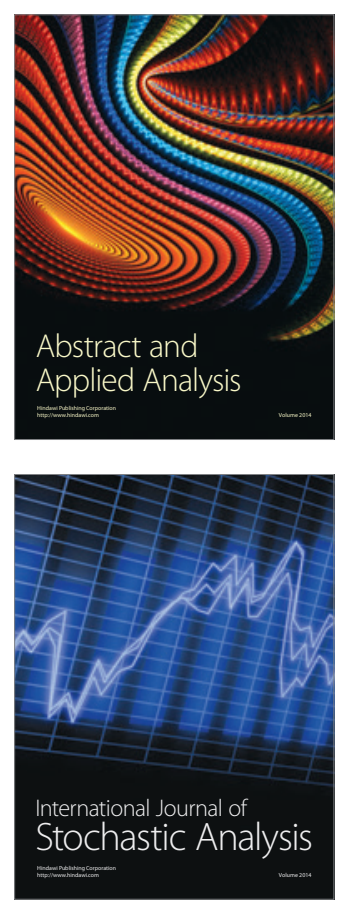

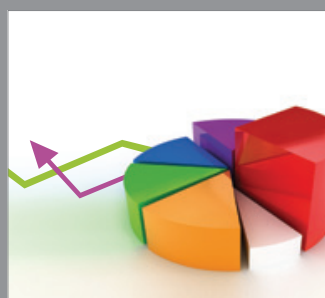

ournal of

Probability and Statistics

Promensencen
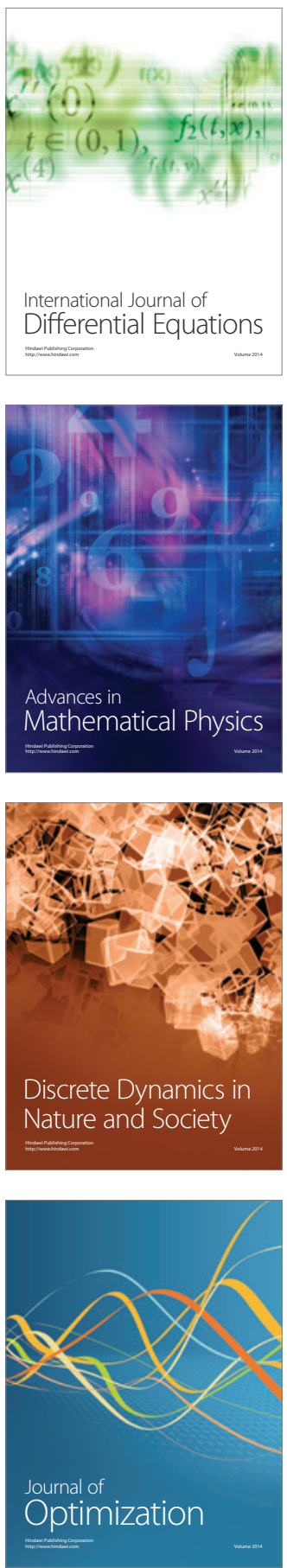